\title{
BMJ Open Patient-controlled hospital admission for patients with severe mental disorders: study protocol for a nationwide prospective multicentre study
}

\author{
Christoffer Torgaard Thomsen, ${ }^{1,2}$ Michael Eriksen Benros, ${ }^{2}$ Lene Halling Hastrup, ${ }^{2,3}$ \\ Per Kragh Andersen, ${ }^{4}$ Domenico Giacco, ${ }^{5}$ Merete Nordentoft ${ }^{2}$
}

To cite: Thomsen CT, Benros ME, Hastrup LH, et al. Patient-controlled hospital admission for patients with severe mental disorders: study protocol for a nationwide prospective multicentre study. BMJ Open 2016;6: 013088 . doi:10.1136/bmjopen-2016013088

- Prepublication history for this paper is available online. To view these files please visit the journal online (http://dx.doi.org/10.1136/ bmjopen-2016-013088).

Received 17 June 2016 Revised 9 August 2016 Accepted 17 August 2016

CrossMark

For numbered affiliations see end of article.

Correspondence to Christoffer Torgaard Thomsen; christoffer.torgaard. thomsen@regionh.dk

\section{ABSTRACT}

Introduction: Patient-controlled hospital admission for individuals with severe mental disorders is a novel approach in mental healthcare. Patients can admit themselves to a hospital unit for a short stay without being assessed by a psychiatrist or contacting the emergency department. Previous studies assessing the outcomes of patient-controlled hospital admission found trends towards reduction in the use of coercive measures and length of hospital stay; however, these studies have methodological shortcomings and small sample sizes. Larger studies are needed to estimate the effect of patient-controlled hospital admission on the use of coercion and of healthcare services.

Design and methods: We aim to recruit at least 315 patients who are offered a contract for patientcontrolled hospital admissions in eight different hospitals in Denmark. Patients will be followed-up for at least 1 year to compare the use of coercive measures and of healthcare services, the use of medications and suicidal behaviour. Descriptive statistics will be used to investigate hospitalisations, global assessment of functioning (GAF) and patient satisfaction with treatment. To minimise selection bias, we will match individuals using patient-controlled hospital admission and controls with a 1:5 ratio via a propensity score based on the following factors: sex, age group, primary diagnosis, substance abuse as secondary diagnosis, coercion, number of psychiatric bed days, psychiatric history, urbanity and suicidal behaviour. Additionally, a historical control study will be undertaken in which patients serve as their own control group prior to index date.

Ethics and dissemination: The study has been approved by The Danish Health and Medicines Authority (j.nr.: 3-3013-934/1/) and by The Danish Data Protection Agency (j.nr.: 2012-58-0004). The study was categorised as a register study by The Danish Health Research Ethics Committee and therefore no further approval was needed (j.nr.: H-2-2014-FSP70). Findings will be disseminated through scientific publications, presentations and in a PhD thesis.

\section{INTRODUCTION}

Patient-controlled hospital admission is a novel approach which follows the concepts of patient-centred care, recovery and empowerment. $^{1} \quad{ }^{2}$ Patient-controlled hospital admission was introduced in Norway in 2005 and enables patients to admit themselves to a designated psychiatric department when in need of care. ${ }^{2}$ The patient and health professionals agree on a contract, which allows the patients to avoid usual admission procedures through the emergency department. This approach was established to increase patient involvement in their care and reduce the use of coercion and length of hospital stay. Despite many efforts to reduce coercion in psychiatry, coercive measures are still widely used and their reduction has been a political priority in Western countries for years. ${ }^{3}{ }^{4}$ Coercion can be defined as any act to which the patient has not given informed consent and should only be applied as a last resort when all other treatment options have failed. ${ }^{5}$ Hence, the use of coercion has been described as an indicator of mental healthcare quality. ${ }^{3} 6$

Historically, provision of mental healthcare has shifted from the hospital to the community with increased availability of outpatient treatment and a decreased number of psychiatric beds. ${ }^{17}$ This has facilitated an increased participation of patients in care and the development of concepts such as patient-centred care, shared decision-making and recovery. ${ }^{1} 8$ However, approaches informed by these concepts have proved difficult to implement in hospital settings and evidence on their outcomes is inconclusive. ${ }^{1} 9$ Psychiatric healthcare services have traditionally used a 'gatekeeper model' according to which the 
health professionals control the admission process. ${ }^{1}$ Usually health professionals make a decision considering the level of care needed and the resources available, for example, number of available psychiatric beds. This procedure may cause stress to some patients with mental illness. Patients may be afraid of being rejected at the emergency department when they only have 'mild' symptoms and this might reduce opportunities for providing preventive care. ${ }^{1}$ Several factors need to be considered by programmes to reduce the use of coercion, such as organisational factors, policy settings, leadership support, patientinvolvement/family-involvement, staff-related factors, treatment factors and culture change, education etc. ${ }^{4}{ }^{10-12}$ Coercion reduction programmes including strategies to address these factors are needed. ${ }^{10}$

A systematic review on patient-controlled hospital admission found six studies, all conducted in Norway including patients with schizophrenia, affective disorders, anxiety disorder, personality disorder and substance abuse. The review showed a tendency towards a reduction in involuntary inpatient care and length of hospital stay. ${ }^{12}$ However, the studies had methodological shortcomings and small sample sizes. The largest study so far has only included 25 patients and did not report any statistically significant results, hence the current evidence is low. ${ }^{9}$ Although no large-scale study has investigated the effectiveness of patient-controlled hospital admission, similar interventions on brief admission have shown promising results, enhancing self-management and preventing crisis in patients with borderline disorders and schizophrenia. ${ }^{13} 14$

\section{AIMS AND OBJECTIVES}

The aim of this prospective cohort study is to evaluate the effectiveness of patient-controlled hospital admission compared to treatment as usual in Denmark. The use of coercion is chosen as a primary outcome based on the findings from Norwegian studies that showed a tendency towards a reduction in the use of coercion. ${ }^{1}$ Secondary outcomes are the use of healthcare services and clinical outcomes such as the use of medications, suicidal behaviour, global assessment of functioning (GAF) and patients' satisfaction with treatment. Furthermore, we will also carry out a historical control study using patients as their own controls and analyse the use of coercion, the use of healthcare services and medications.

A. Primary research question:

Do patient-controlled hospital admissions reduce the use of coercion compared to treatment as usual?

B. Secondary research questions:

Regarding the use of healthcare services:

- Are there differences in the length of hospital stay and number of admissions between patients' enrolled in patient-controlled hospital admission and treatment as usual?

- What is the mean length of hospital stay among cases?
- During which period is patient-controlled hospital admission frequently used and how often is patientcontrolled hospital admission converted to treatment as usual?

- How often are patient-controlled beds occupied and for how long?

Regarding clinical outcomes:

- Are there differences in the use of medications between cases and controls?

- Are incidences of deliberated self-harm more frequent in either group?

- What is the global assessment of functioning among cases?

- What is the satisfaction with treatment among cases? We hypothesise that:

Psychiatric patients with a contract to a patient-controlled hospital admission with the ability of self-referral to a designated unit will receive less coercive measures compared to those receiving treatment as usual.

\section{METHODS AND ANALYSIS}

\section{Study design}

In this matched prospective cohort study we compare patients in the patient-controlled hospital admission group (expected $n=315$ ) with those who were receiving treatment as usual (figure 1). Patients with a contract to patient-controlled hospital admission are intended to be followed-up for at least 1 year from the beginning of the study period (defined as the date the contract is signed, the index date). Patients are then followed until death, emigration from Denmark or until the end of study period (August, 2016). All psychiatric inpatients born in Denmark between 1 January 1937 to 3 September 1998 and alive at the index date during the study period (2013-2016) are identified and followed from the beginning of The Danish Psychiatric Central Register in 1969. Patients with a contract will be compared with one matched, 'unexposed' control group identified in the registers (=index date=matching date) via a propensity score model as described below. Controls must be living in Denmark and be alive at the index date. Strengthening the Reporting of Observational Studies in Epidemiology (STROBE) guidelines will be followed in the manuscript. ${ }^{15}$

\section{Settings}

The study was initiated by the Danish Health Authority, which is responsible for the overall project management in collaboration with representatives from each of the five counties in Denmark. Clinical data will be obtained from the following seven cities where patient-controlled hospital admission is implemented (number of patientcontrolled beds): Copenhagen and Hillerød (7), Vordingborg (3), Odense (3), Svendborg (1), Aarhus (5) and Brønderslev (2) (figure 2). The Research unit at Mental Health Centre Copenhagen, Frederiksberg and Biostatistics Department, Copenhagen is responsible 


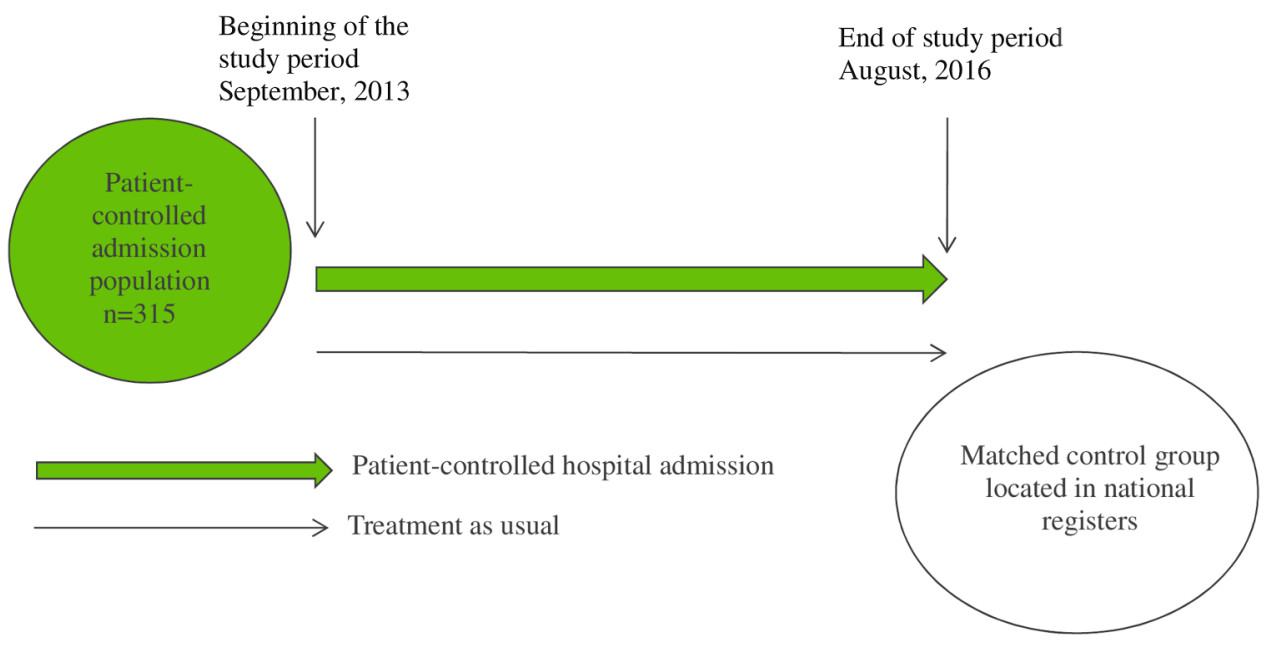

Figure 1 Matched controlled study design.

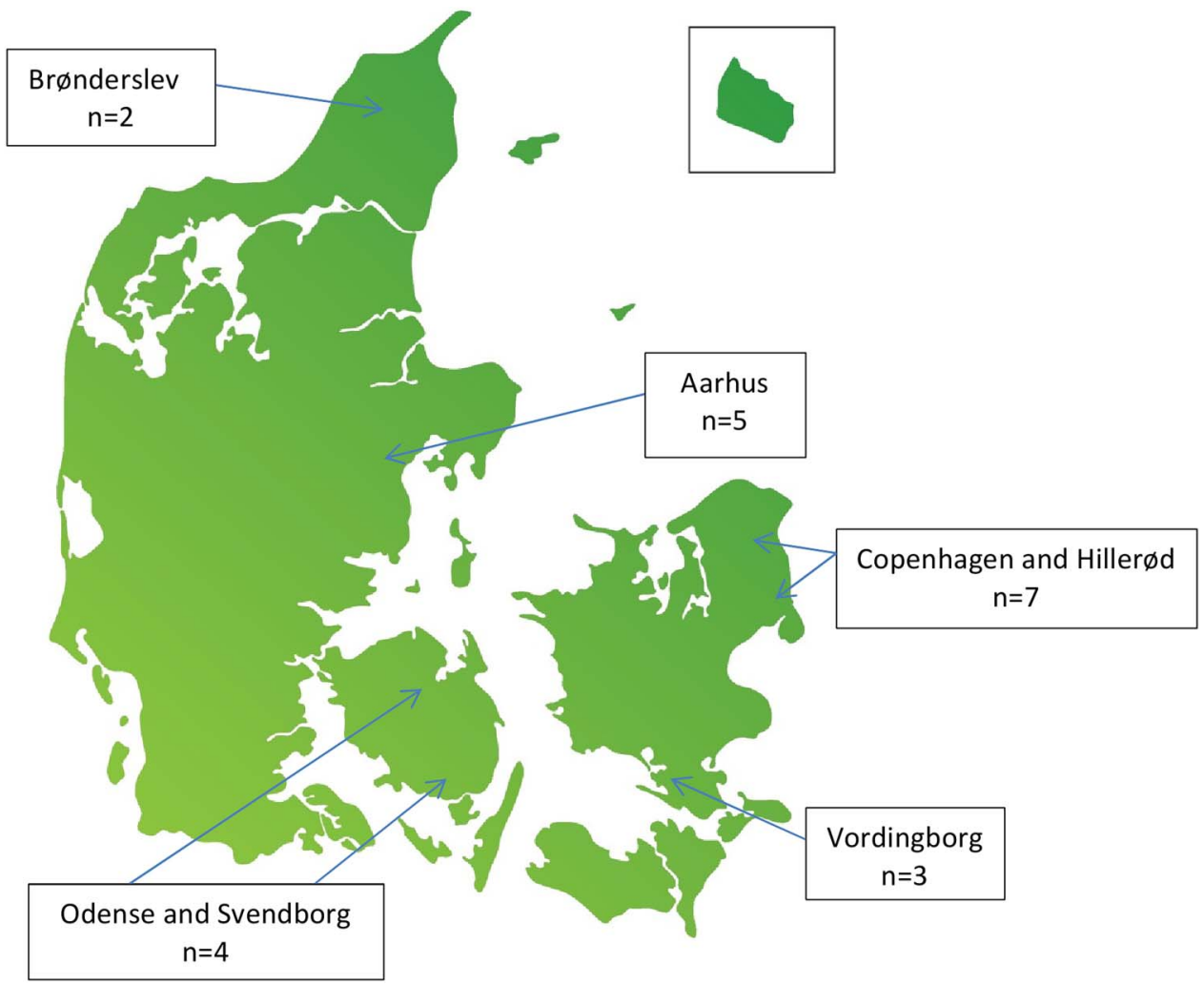

Figure 2 Distribution and number $(n)$ of patient-controlled beds in Denmark.

for data extraction from nationwide registers, which is expected to be undertaken in August 2016. All treatments in Danish psychiatric hospitals are free of charge for all residents and the designated patient-controlled hospital beds have $100 \%$ financing no matter how much they are used by the patients in the programme.

\section{Participants}

Inclusion criteria:

Adult patients with mental disorder(s) who, according to the assessment of their healthcare professionals could profit from patient-controlled hospital admission.
Exclusion criteria:

Lack of informed consent.

\section{Procedures}

Patient-controlled hospital admission is offered in eight different psychiatric hospitals in Denmark. The common denominator for all regions is that patients are assigned to patient-controlled hospital admission for a short period of stay. Eligible patients will be identified by healthcare professionals (usually psychiatrists and nurses) in hospital or outpatient settings. A written contract is signed by the patients and their healthcare 
professionals in which the principles and the rules of patient-controlled hospital admission are outlined. This contract allows patients to self-refer to a designated hospital department, intendedly before serious deterioration of illness and without objection from the clinical staff. Typically, the patient will contact the unit by telephone to check availability and set up an appointment. In case no beds are available, the clinical staff will recontact the patient later, once admission is possible. No fixed treatment procedures are followed. Once admitted the patient's needs for support, rest and structure and their desires and individual needs are sought to be met as far as possible within the resources of the hospital. If additional treatment is needed the patient is converted to a conventional hospitalisation. Most counties allow in-patient stay for patient-controlled hospital admission for a maximum 5 days. Only one hospital has a maximum hospitalisation of 7 days. There are small differences in contact hours for admission between units and some hospitals have implemented quarantine after admission as well as limited number of admissions during a year.

\section{Data collection}

The study started in September, 2013. Collaborating units (see figure 2) are responsible for data recordings (see figure 2). A template for data collection is used to ensure that data on personal identification number, contract date, Client Satisfaction Questionnaire (CSQ) and GAF is collected. Hard copies of waiting lists for a patient-controlled hospital admission, CSQ and GAF will be stored in a locked cabinet. Data will not be available for extraction or analysis in national registers before August 2016.

\section{Registers}

Citizens in Denmark are assigned a unique 10-digit identification (ID) number, enabling accurate linkage between nationwide registers of sociodemographic and health-related variables described in the following five registers. (1) Since 1968, The Danish Civil Registration System (CRS) has provided information on the identity and vital status of all individuals living in Denmark. ${ }^{16}$ (2) The Danish Psychiatric Central Register (PCRR) covers information on all psychiatric admissions in Denmark since 1 April 1969. ${ }^{17}$ From 1 January 1994, the International Classification of Disease, 10th Revision (ICD-10) classification was introduced in Denmark. ${ }^{17}$ (3) National Registry of Patients (NRP) covers data on all individuals in Danish somatic hospitals since 1977 and has been expanded over the years also covering psychiatric information. ${ }^{18}$ (4) Register of National Coercive Measures in Psychiatric Treatment is a register of all recordings of used coercion in Denmark between 1999 and 2016, since all psychiatric inpatient facilities in Denmark are legally mandated to report every coercive measure applied. $^{19}$ (5) The Danish Register of Medicinal Product Statistics comprises information on all prescriptions collected outside hospitals since 1 January $1994 .^{20}$

\section{Baseline measures}

The following data will be collected at baseline (index date):

- Demographic characteristics: age and sex.

- Psychiatric diagnosis according to the ICD-10. ${ }^{21}$ The primary diagnosis is obtained from the registers at discharge from the hospitals.

- Duration of illness measured in years.

- Previous coercion 1 or 2 years before index date (any coercive measurements, compulsory admission/involuntary detainment, restraint and forced medication).

- Healthcare consumption in the year before contract for patient-controlled admission: number of admissions and length of hospital stay.

- Medication use closest to index date.

- Deliberated self-harm 1 year prior to index date.

- Severity of illness rated by health professional using GAF. The first register GAF measurement is used after index date.

\section{Follow-up measures}

In order to evaluate patient-controlled hospital admission, cases will be followed for at least 1 year according to the following outcomes.

\section{Assessment of coercive measures}

The primary outcome is any coercive measure. Coercive measures are defined as compulsory admission, involuntarily detention, restraint and forced treatment. According to the Danish Mental Health Act, formal coercion is defined as any act to which the patient has not given informed consent. ${ }^{5}$

Compulsory admission or involuntary detention can be applied if the person is psychotic or in a state similar to being psychotic and at the same time is (1) of danger to himself/herself or other or (2) the patient is so severely mentally ill that his/her condition will deteriorate substantially without treatment. ${ }^{5}$ Restraint is defined and divided into the following five groups. (1) Fixation by a mechanical device, which includes immobilisation with leather belts around the waist and/or wrist and ankle cuffs. Gloves are also used, but rarely. (2) Being held by staff. (3) Locking a door of an open ward. (4) Administration of acute sedative medication. (5) Shielding patients for more than 24 hours. Forced treatment is defined as psychopharmacological medication, ECT, involuntary diet intake and assisted outpatient treatment. In-patient coercion, such as acute sedative medication, may be applied even though a patient is admitted voluntarily. The use of seclusion is generally forbidden by the law in Denmark except at one forensic psychiatric facility. ${ }^{5}$ 


\section{Assessment of hospitalisation pattern}

To distinguish conventional hospitalisation from patientcontrolled hospital admissions in the registers, codes AAF12 and AAF13 were made for admission and discharge, respectively. Number of admissions and length of in-patient stay are measured and compared between the two approaches.

\section{Assessment of medication}

Prescriptions for selected antipsychotics (ACT code N05A), antidepressants (ACT code N06A) and benzodiazepines (ACT codes N03AE, N05BA, N05CD and N05CF) use are identified for each individual and controls at index date and followed-up the preceding year. ${ }^{22}$ For each prescription we will identify the Anatomic Therapeutic Classification (ACT) codes and defined daily dose (DDD) as well as the date of purchase for each medication.

\section{Assessment of deliberated self-harm}

Deliberated self-harm is defined using the following ICD-10 codes: X60-84. However, since deliberate selfharm is under-recorded in the Danish registers, additional definitions of probable self-harm were added. The first definition of probable self-harm, covers injuries to the lower forearm and accidental poisonings in individuals diagnosed with mental disorders, defined as an ICD-10 main diagnosis F00-F99 in combination with subdiagnosis (S51, S55, S59, S61, S65, S69, T36-T50, T52-T60) or any main diagnosis (T39, T40 (except T401), T43, T58). ${ }^{23}$ The second and broader definition covering probable self-harm, also includes accidental poisonings and selected injuries to the forearm categorised as S51, S55, S59, S61, S65, S69, T36-T60 (exceptT401), X40-X49, X60-X84, and Y10-Y34 in ICD-10. ${ }^{23}$

\section{Descriptive analysis among cases}

\section{Assessment of hospitalisation pattern}

We aim at measuring number of times hospital treatment is converted to standard care during patientcontrolled hospital admission and how often beds are occupied, together with mean number of days a patient had to wait for admission in the intervention. Furthermore we will study variation in service use. This will be measured during a limited time period in 2013-2016.

\section{Assessment of GAF}

The GAF score is a numeric scale from 1 to 100 . The GAF will be used to measure the psychosocial functioning level among cases. ${ }^{24} \mathrm{~A}$ higher score indicates a better functioning level. GAF is measured at least one point in time during the study period. In Copenhagen and Hillerød, the GAF score is assessed at discharge. In all other counties the GAF score is assessed when the contract is renegotiated or ended. In all study centres
GAF-F is used, with the exception of the hospital in Hillerød using Global GAF.

\section{Assessment of CSQ-8}

CSQ is used to measure satisfaction with mental healthcare and is used in Copenhagen, Hillerød and Svendborg. ${ }^{25}$ CSQ-8 is measured at least one point in time during the contract period. Scores range from 4 to 32 , with higher values indicating higher satisfaction with care.

\section{Sample size calculation}

An average of $20 \%$ of patients in contact with the Danish psychiatric hospitals is subjected to coercion. We expect that this estimate is conservative for our sample which includes cases with severe mental illness. Including a minimum of 315 patients in the study and five matched controls for each patient, we will be able, with a power of $90 \%$ and a statistical significance level of $5 \%$ to detect a difference of $20 \%$ versus $30 \%$ who is subjected to coercion during a year. A larger number of patients will make it possible to analyse specific coercive measures.

\section{Analysis}

A propensity score logistic regression model, that is, a model where the outcome is signing a contract versus not signing a contract, will be developed to adjust for the following factors: age, sex and mental disorder classified according to the following main diagnoses closest to index date: Organic mental disorder (ICD-10: F00-F09), substance abuse (ICD-10: F10-19), schizophrenia disorder (ICD-10: F20-F29), affective disorder (ICD10: F30-39), anxiety disorder (ICD-10: F40-48), behavioural syndromes (ICD-10: 50-59); personality disorder (ICD-10: 60-69), mental retardation (ICD-10: 70-79), developmental disorder (ICD-10: 80-89), attentiondeficit hyperactivity disorder (ICD-10:90-98) or others defined as diagnoses other than those listed above, ${ }^{21}$ substance abuse as secondary diagnosis, any coercive measures during the past 2 years prior to index date, number of psychiatric bed days in the year before the matching date, previous suicidal behaviour (before index date), family history of mental disorders defined as at least one parent diagnosed with any mental disorder (ICD-8 or ICD-10). An exact matching is chosen on sex, psychiatric disorder and substance abuse as secondary diagnosis. We further aim at matching for urbanity (rural, province and capital) or hospital location if feasible. Using greedy nearest neighbour matching algorithms we will match five controls to each patient with a contract based on the estimated propensity score. The outcome variables (coercion, the use of healthcare services, medication use and suicide behaviour) will be analysed taking the length of the follow-up period into account and using robust SEs. Further analyses in which patients serve as their own control group 1 year prior to the index date will be 
performed. Simple descriptive analysis will be undertaken to investigate patients' satisfaction with treatment, GAF and hospitalisation pattern in regard to how often beds are occupied, together with mean number of days a patient had to wait for admission. Statistical Analysis System (SAS) software will be used throughout the analysis.

\section{Strengths and limitations of this study}

To the best of our knowledge this study will be the largest cohort study to compare patient-controlled admission with treatment as usual among patients with severe mental illness. It is a nationwide multicentre study that will involve hospitals in seven cities distributed in five counties in Denmark. This study is likely to advance knowledge and improve practice. Even negative results on outcomes will be of great value when trying to develop new treatment strategies for patients with severe mental illness. The quality of evidence in previous studies on patient-controlled admission has been rated as low or very low according to the Oxford Centre for Evidence-Based Medicine classification. ${ }^{1}$ Furthermore, models favouring patient participation in care have proven difficult to implement in hospital settings. ${ }^{1}$ This observational study contributes to the ongoing research of user involvement by increasing the quality of evidence compared to previous studies and investigates patientcontrolled hospital admission in inpatient settings. The nationwide registers in Denmark are of high quality and the law in Denmark requires registration of the episodes of coercion in these registers, hence we expect that the information collected on the primary outcome will be highly reliable.

Being a cohort study, patients will not be randomised to either approaches and inclusion criteria are based on the healthcare professional's judgement. In order to minimise selection bias, we will match controls on selected patients characteristics. Furthermore, similarly to most observational studies there may be attrition during the study period, which might affect data collection. The organisation and provision of mental healthcare differ to some extent among counties. For instance, some departments have different contact hours. Quarantine after admission has been implemented in some hospitals to avoid the misuse of services as well as a limited number of days a bed can be used and number of patient-controlled hospital admissions in a year. One department has two patient-controlled beds located in one room.

\section{ETHICS}

The Danish Health Research Ethics Committee has ethically cleared on 18 September 2014 and no further approval is needed; hence the investigation is categorised as a register study (Protocol nr.: H-2-2014-FSP70). All personal information from the registers is anonymised when used for research purposes. Participants have the right to withdraw consent from the study at any time without consequences for their future care or treatment.

\section{DISSEMINATION}

Investigating the effect of patient-controlled hospital admission in mental healthcare services will contribute to the scarce evidence level in this field. ${ }^{1}{ }^{2}{ }^{26}$ Despite the differences in national laws, organisational structure for healthcare and mental health systems in different countries and areas, our findings are expected to inform implementation of patient participation strategies in mental healthcare services. We will disseminate our results through scientific papers, presentations and in a $\mathrm{PhD}$ thesis and outline recommendations for mental health professionals.

\section{Author affiliations}

${ }^{1}$ Mental Health Centre Frederiksberg, Copenhagen University Hospital, Copenhagen, Denmark

${ }^{2}$ Mental Health Centre Copenhagen, Copenhagen University Hospital, Copenhagen, Denmark

${ }^{3}$ Psychiatric Research Unit, Region Zealand, Slagelse, Denmark ${ }^{4}$ Department of Biostatistics, University of Copenhagen, Copenhagen, Denmark

${ }^{5}$ Unit for Social and Community Psychiatry (World Health Organisation, Collaborating Centre for Mental Health Services Development), Queen Mary, University of London, London, UK

Acknowledgements The authors would like to thank the head of the Mental Health Centre, Frederiksberg, Dr Kristen Kistrup for making the research possible. The authors thank all health professionals affiliated to the project in the five counties and their dedicated work on registration and data collection and believing in the project.

Contributors CTT, MN, MEB and PKA, contributed to the design of the study. LHH has made substantial contribution to the project. MEB, LHH and DG have participated in revising the manuscript critically for important intellectual content. CTT drafted the manuscript and all authors received and approved the final manuscript.

Funding This research is supported by the Danish Ministry of Health and Mental Health Centre, Frederiksberg.

Competing interests None declared.

Ethics approval The study was approved by The Danish Data Protection Agency on 12 January 2015 (J.nr.: 2012-58-0004) and The Danish Health and Medicines Authority on 212015 (Case nr. 3-3013-934/1/).

Provenance and peer review Not commissioned; externally peer reviewed.

Open Access This is an Open Access article distributed in accordance with the Creative Commons Attribution Non Commercial (CC BY-NC 4.0) license, which permits others to distribute, remix, adapt, build upon this work noncommercially, and license their derivative works on different terms, provided the original work is properly cited and the use is non-commercial. See: http:// creativecommons.org/licenses/by-nc/4.0/

\section{REFERENCES}

1. Strand M, von Hausswolff-Juhlin Y. Patient-controlled hospital admission in psychiatry: a systematic review. Nord $J$ Psychiatry 2015;69:574-86.

2. Heskestad S, Tytlandsvik M. [Patient-guided crisis admissions for severe psychotic conditions]. Tidsskr Nor Laegeforen 2008;128:32-5.

3. Kalisova L, Raboch J, Nawka A, et al. Do patient and ward-related characteristics influence the use of coercive measures? Results from 
the EUNOMIA international study. Soc Psychiatry Psychiatr Epidemiol 2014:49:1619-29.

4. Husum TL, Bjorngaard JH, Finset A, et al. A cross-sectional prospective study of seclusion, restraint and involuntary medication in acute psychiatric wards: patient, staff and ward characteristics. BMC Health Serv Res 2010;10:89.

5. Danish Health Authority. Anvendelse af Tvang i Psykiatrien 2008. Copenhagen, Denmark: The Danish Health Authority, 2010:1-16.

6. Steinert T, Lepping $P$, Bernhardsgrutter $R$, et al. Incidence of seclusion and restraint in psychiatric hospitals: a literature review and survey of international trends. Soc Psychiatry Psychiatr Epidemiol 2010;45:889-97.

7. Nordentoft M, Pedersen MG, Pedersen CB, et al. The new asylums in the community: severely ill psychiatric patients living in psychiatric supported housing facilities. A Danish register-based study of prognostic factors, use of psychiatric services, and mortality. Soc Psychiatry Psychiatr Epidemiol 2012;47:1251-61.

8. Olso TM, Gudde CB, Moljord IE, et al. More than just a bed: mental health service users' experiences of self-referral admission. Int J Ment Health Syst 2016;10:11.

9. Storm M, Edwards A. Models of user involvement in the mental health context: intentions and implementation challenges. Psychiatr Q 2013;84:313-27.

10. Bak J, Brandt-Christensen M, Sestoft DM, et al. Mechanical restraint-which interventions prevent episodes of mechanical restraint?- a systematic review. Perspect Psychiatr Care 2012;48:83-94.

11. Gaskin CJ, Elsom SJ, Happell B. Interventions for reducing the use of seclusion in psychiatric facilities: review of the literature. Br J Psychiatry 2007;191:298-303.

12. Flammer $\mathrm{E}$, Steinert $\mathrm{T}$, Eisele $\mathrm{F}$, et al. Who is subjected to coercive measures as a psychiatric inpatient? A multi-level analysis. Clin Pract Epidemiol Ment Health 2013;9:110-19.

13. Helleman M, Goossens PJ, Kaasenbrood A, et al. Evidence base and components of Brief Admission as an intervention for patients with borderline personality disorder: a review of the literature. Perspect Psychiatr Care 2014;50:65-75.
14. Little J, Stephens D. A patient-based voucher system of brief hospitalisation. Aust N Z J Psychiatry 1999;33:429-32.

15. STROBE Statement. Secondary STROBE Statement 2007. http:// strobe-statement.org/index.php?id=available-checklists

16. Pedersen CB. The Danish Civil Registration System. Scand J Public Health 2011;39(7 Suppl):22-5.

17. Mors O, Perto GP, Mortensen PB. The Danish Psychiatric Central Research Register. Scand J Public Health 2011;39(7 Suppl):54-7.

18. Lynge E, Sandegaard JL, Rebolj M. The Danish National Patient Register. Scand J Public Health 2011;39(7 Suppl):30-3.

19. Sundhedsdatastyrelsen. Register over Anvendelse af Tvang $i$ Psykiatrien. 2016. http://www.esundhed.dk/

20. Kildemoes HW, Sorensen HT, Hallas J. The Danish National Prescription Registry. Scand J Public Health 2011;39(7 Suppl): 38-41.

21. World Health Organization. The ICD-10 classification of mental and behavioural disorders: clinical descriptions and diagnostic guidelines. WHO, 1992.

22. WHO Collaborating Centre for Drug Statistics Methodology, Guidelines for ATC classification and DDD assignment 2015. Oslo, 2015. Secondary WHO Collaborating Centre for Drug Statistics Methodology, Guidelines for ATC classification and DDD assignment 2015. Oslo, 2015. 2016. http://www.whocc.no/atc_ddd_publications/ guidelines

23. Erlangsen A, Lind BD, Stuart EA, et al. Short-term and long-term effects of psychosocial therapy for people after deliberate self-harm: a register-based, nationwide multicentre study using propensity score matching. Lancet Psychiatry 2015;2:49-58.

24. Abe T, Ikeda K, Kuroda K, et al. Assessment of psychiatric outcomes in Japan based on diagnostic procedure combination information. Psychiatr Q 2011;82:163-75.

25. Attkisson CC, Zwick R. The client satisfaction questionnaire. Psychometric properties and correlations with service utilization and psychotherapy outcome. Eval Program Plann 1982;5:233-7.

26. Richards T, Montori VM, Godlee F, et al. Let the patient revolution begin. BMJ 2013;346:f2614. 